\title{
Some issues related to the forecast of strong ground motion fields
}

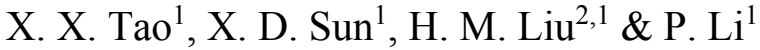 \\ ${ }^{1}$ Harbin Institute of Technology, China \\ ${ }^{2}$ Chongqing Communications Research \& Design Institute, China
}

\begin{abstract}
In conjunction with a companion paper by the same authors in this volume, this paper discusses the issues arising in the process of forecasting strong motion fields that need to be dealt with further. Firstly, the field must be estimated from the same source model, rather than taking the average motion at a site from more than 30 source models as in the current seismic hazard assessment practice. The second issue concerns the random synthesis, that is, the uncertainty in the envelop of motion time histories from a sub source must be treated in the same way for all ground points rather than vary randomly as in the current practice. The third concerns the uncertainty in the rupture velocity, which must not be changed randomly in the synthesis. Finally, the fourth issue concerns the order of the filters and the integrations of accelerations and velocities of the high and low frequency motions before being combined together. Through a sensitivity analysis, the consequences of the above issues are presented and further study is suggested.

Keywords: forecast, ground motion field, source model, uncertainty, filter and integration.
\end{abstract}

\section{Introduction}

A set of approaches for forecasting strong ground motion at engineering sites have been developed in recent years [1-6] and are applied for disaster reduction in some engineering projects and urban areas [7-9]. For large city or long span structures, the ground motion field is required for seismic analysis. There are some issues that need to be dealt with, if the known approaches for forecasting the field are applied, from the authors' point of view. Four among these issues are discussed in this paper. 
74 Earthquake Ground Motion: Input Definition for Aseismic Design

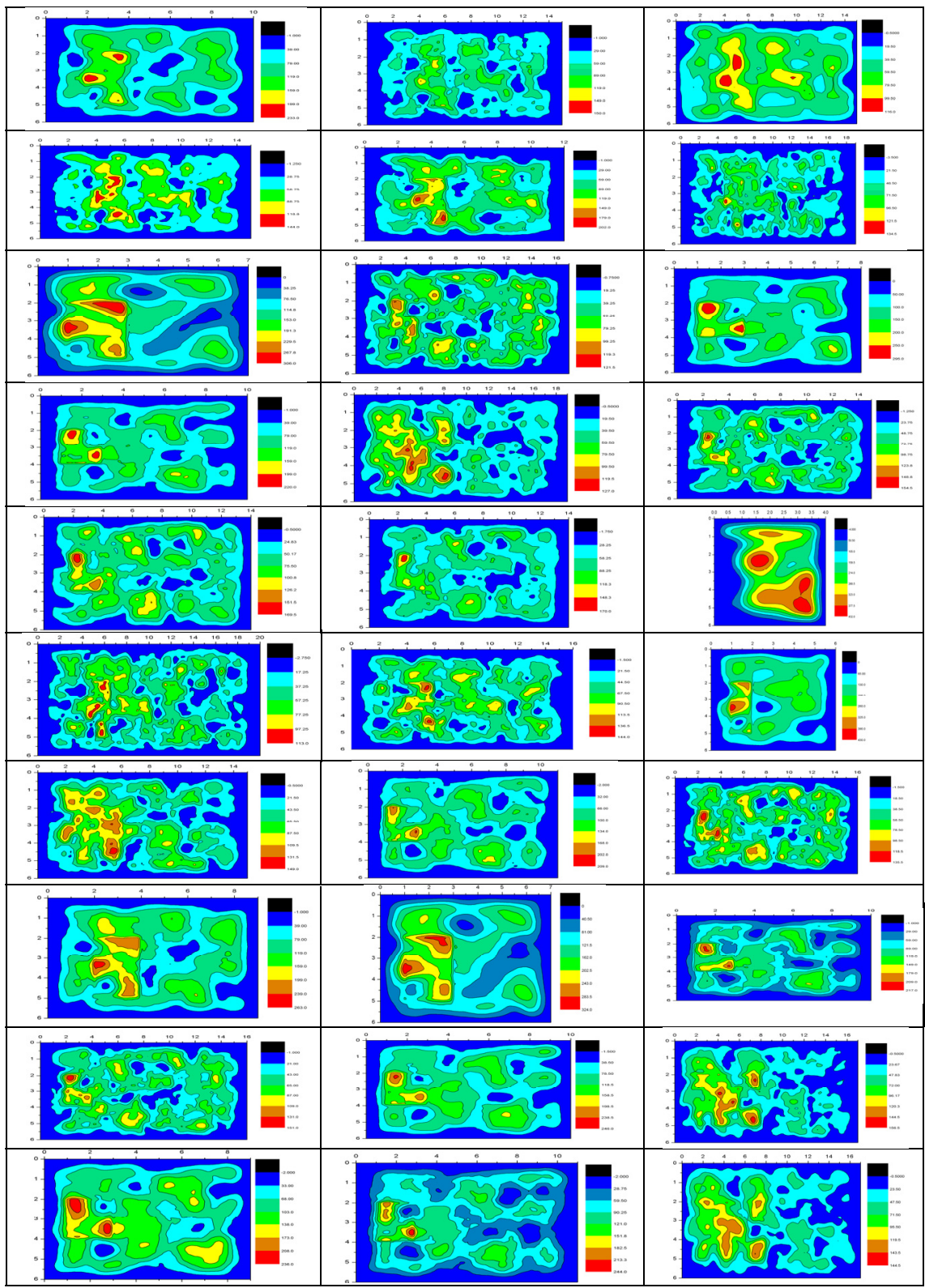

Figure 1: $\quad$ Source models for an earthquake with magnitude 6.0. 


\section{Finite fault source model}

A finite fault source model is needed to characterize the ground motion due to a large magnitude earthquake in the near field, for otherwise the motion tends to be overestimated while the directivity and the hanging wall effect cannot be simulated. This model allows the representation of a realistic inhomogeneous distribution of the dislocation on the rupture plane. In the model, the faulting plane is divided into many sub-sources and the specified dislocation energy is assigned to each one of them as point source. The most difficult issue at present is to determine the source parameters that describe the dynamics of the slip, such as stress drop and rise time and the inhomogeneous distribution of the dislocation on the fault plane. In the hybrid finite fault source model adopted by Tao and Wang [6], the slip distribution on the fault plane is determined by the superposition of a long wave length slip from an asperity model [3] for the physical nature and a short wave length slip from a $k$ square model [10] for the uncertainty. In order to take into account this uncertainty and that in the parameter estimation of the source size and the average slip, 30 source models at least are required for each site in the current seismic hazard assessment practice. Fig. 1 shows 30 models for an assessment of a site in southeastern China, for example. It can be noted that there are very big differences between each pair of models. Therefore the ground motions generated from them must be different.

Fig. 2 shows 30 acceleration response spectra at a ground point from the source models of fig. 1. In that figure, the scattering degree of the spectra from their mean can be noted. In practice, the average spectrum and the ground motion time history corresponding to the spectrum closest to it are then selected as input for seismic analysis.

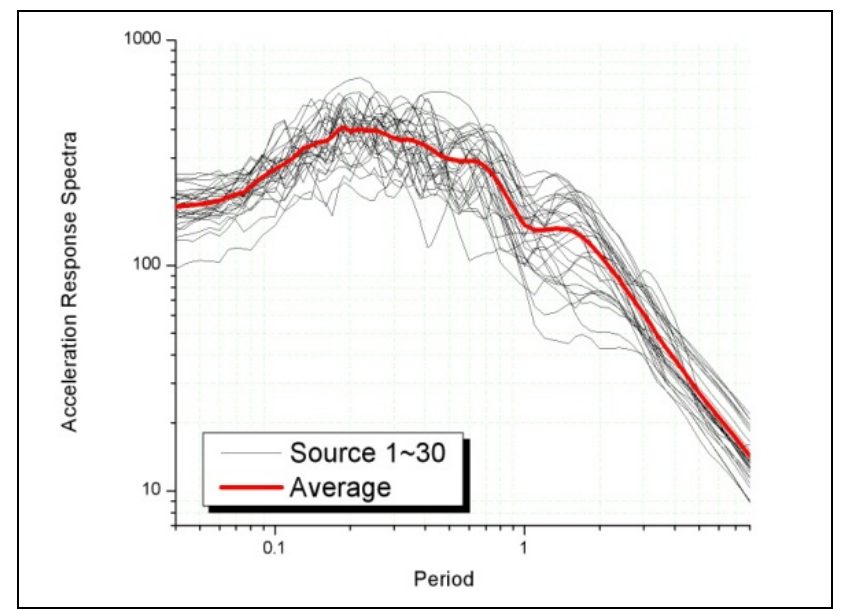

Figure 2: Response spectra at a point from 30 models and their mean. 
It is obvious that the selected time histories at different points may not come from the same source model, if this approach is applied to forecast a motion field. The spatial pattern of the obtained field must be distorted by this difference. The authors like to raise the issue that just one source model must be selected for motion field forecasting and the way to do that must be dealt with care in the future.

\section{Uncertainty in high frequency motion synthesis}

In the random synthesis of high frequency motion, the acceleration time history at a site is obtained by summing those of all sub-sources, as follows:

$$
a(t)=\sum_{i=1}^{N_{L}} \sum_{j=1}^{N_{W}} a_{i j}\left(t+\Delta t_{i j}\right)
$$

Time history is obtained from the spectrum by inverse Fourier transform. The Fourier amplitude spectrum caused by each sub-source is obtained from the equation $[4,11]$

$$
F A\left(M_{0}, f, R\right)=S\left(M_{0}, f\right) G(R) D(R, f) A(f) P(f)
$$

Then, a phase spectrum is added which is generated randomly with uniform distribution in the range $(0,2 \pi)$. Fig. 3 shows 30 response spectra at a point from 30 phase spectra and a given source model.

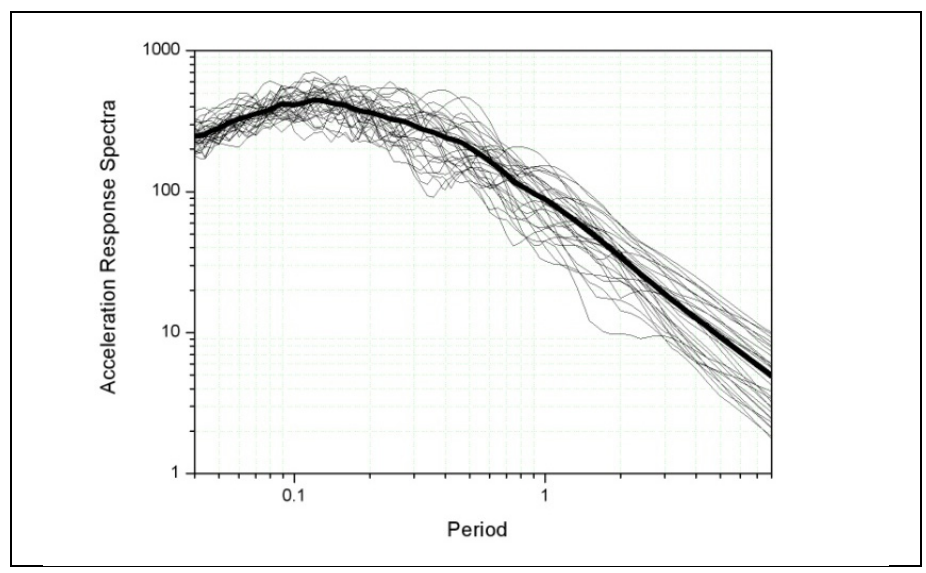

Figure 3: Response spectra at a point from 30 random phase spectra and their mean. 
It can be noted also in that figure that there is a non-negligible scatter of the spectra from their mean. This means that random scattering of spectra should be inferred from the randomly generated phase spectra. In engineering practice, the mean spectrum is selected. The spatial pattern of the forecasted motion field must be distorted by this uncertainty. Therefore, the issue of phase spectrum uncertainty must be resolved for the forecast of a strong ground motion field.

The $\Delta t_{i j}$ in eqn (1) is the time delay from the triggering time of the $i j^{\text {th }}$ sub-source and the travel time between the sub-source to the site. The former depends on the sub-source position on the fault plane and the rupture velocity. Some researchers suggested taking into account the uncertainty in the rupture velocity by adding to the mean velocity or subtracting from it a small random difference $[12,13]$. In order to see the effect of this treatment, 30 response spectra at a point are calculated from a given source model and the same phase spectrum with the changeable rupture velocity. Fig. 4 shows the result.

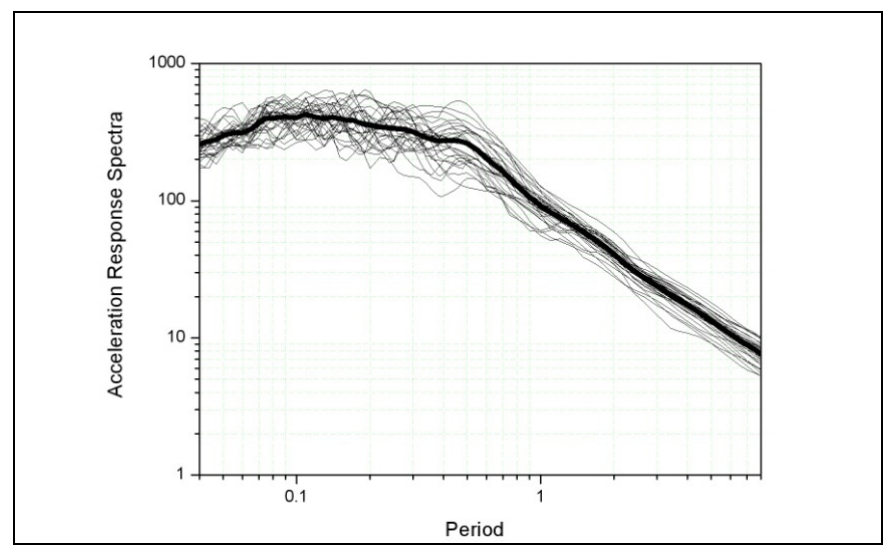

Figure 4: Response spectra at a point from a given source model and the same phase spectrum with changeable rupture velocity.

It can be seen from that figure that the uncertainty in rupture velocity is also non-negligible in the forecast of ground motion field. The authors suggest that it seems better to keep the same velocity rather than to adopt a randomly variable one in the field synthesis.

\section{Filtering before the superposition of motions}

In order to overcome the disadvantages of the above synthesis in the low frequency range, the obtained motion is further superposed in the time domain to a motion obtained from a numerical approach, such as the finite element or finite difference method, after being high pass or low pass filtered, respectively. Usually, the synthesized motion is an acceleration time history, and the motion from numerical calculation is displacement. In general, the final result is required as acceleration, velocity and displacement time histories. There are two ways to obtain filtered 
low frequency motion. One is to differentiate the calculated low frequency displacement to get the velocity and then the acceleration, and finally to filter the three time histories. The other is to filter the displacement, and then to differentiate the result to obtain velocity and acceleration. Fig. 5 shows the results of these two procedures.

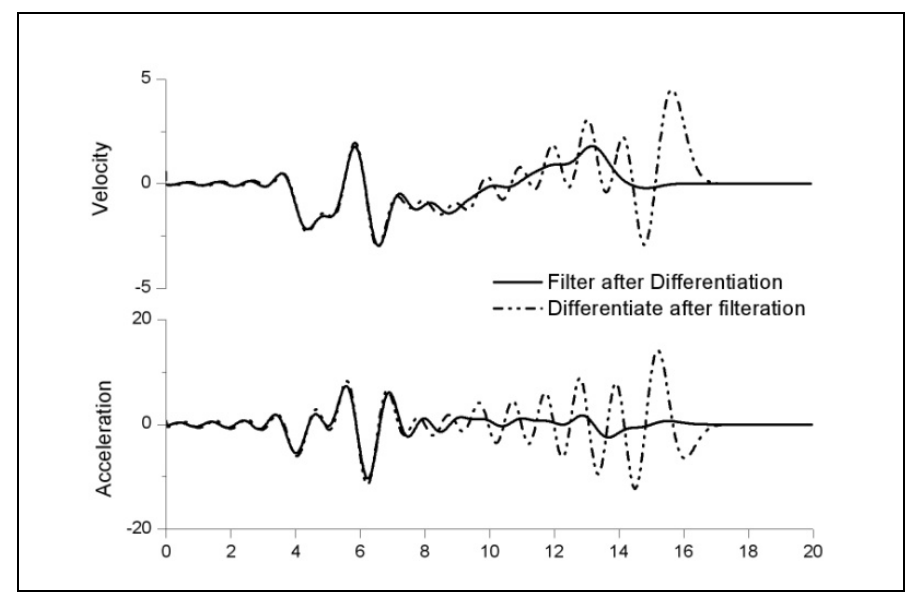

Figure 5: Velocity and acceleration by two differential and filtering procedures from a given displacement time history.

It is obvious from that figure that the time histories are the same in the first $7 \mathrm{~s}$ and there is a terrible numerical noise in the form of high frequency oscillation in the result by the second procedure. This means that the differentiation must be performed before the filtering.

There are also two ways to integrate and to filter the synthesized high frequency acceleration time history. One is to filter the acceleration first, then to integrate to obtain the velocity and integrate again to obtain the displacement. The other is to perform the integrations to obtain the velocity and displacement first, then to filter all three time histories. Fig. 6 shows the results of these two procedures.

It can be seen from the figure that the two velocity time histories are almost the same, while there is an obvious very slow shift with time in the displacement time history by the first procedure. That means that the filtering must be performed after integration.

In summary, filtering must be performed at the end in both situations. Therefore, the last issue raised in this paper is the order of the integration or differentiation and the filtering of time histories.

\section{Conclusions}

Four issues arising in the forecast of a ground motion field are discussed in this paper. The uncertainty involved in each of them brings about a significant effect on the final result. 


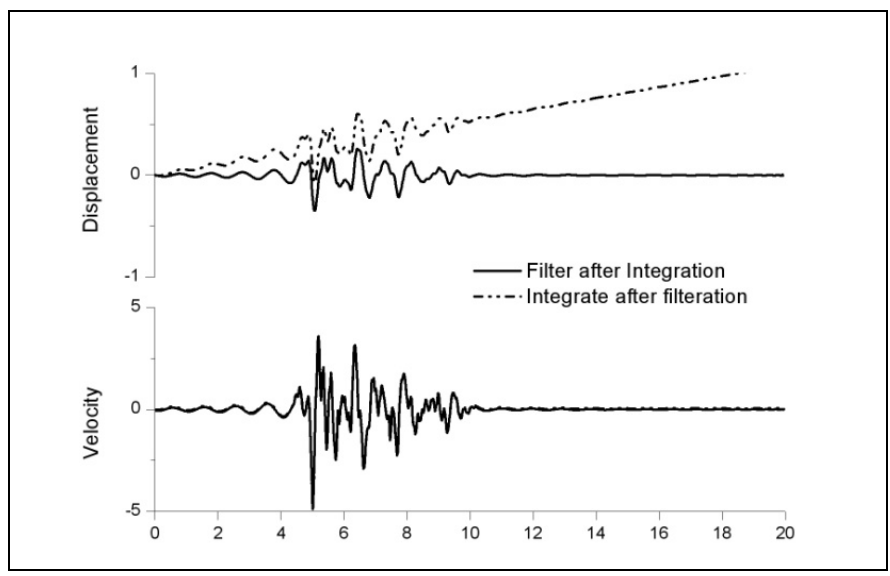

Figure 6: Velocity and displacement by two integrate and filtering procedures from a given acceleration time history.

Fig. 7 shows the deviations in the spectra generated from differences in source models, randomness of phase spectra and variations in rupture velocity relative to its mean. It can be observed in this figure that the effect of phase spectra randomness is the most pronounced and it is getting notably larger with period; that of source model differences is in the middle among the three, while that of variation in rupture velocity is the weakest and is getting quite small for periods greater than $1.0 \mathrm{~s}$.

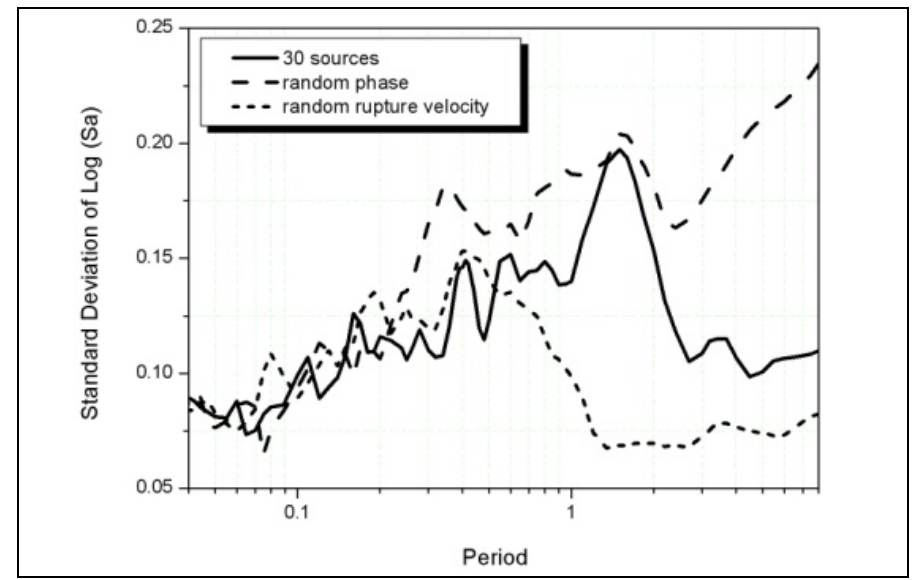

Figure 7: Deviations of logarithm of spectral amplitudes due to different source model, random phase spectra and variation in rupture velocity.

The importance of the order of integration or differentiation and filtering is also emphasized. The filtering must be performed after the integration or differentiation. 


\section{References}

[1] Hanks, T.C. \& McGuire, R.K., The character of high-frequency strong ground motion. Bulletin of the Seismological Society of America, 71(6), pp. 2071-2095, 1981.

[2] Beresnev, I.A. \& Atkinson, G., Stochastic finite-fault modeling of ground motions from the 1994 Northridge, California, earthquake, I. Validation on rock sites. Bulletin of the Seismological Society of America, 88(6), pp. 1392-1401, 1998.

[3] Somerville, P.G., Irikura, K., Graves, R.W., Wald, D., Abrahamson, N., Iwasaki, Y., Kagawa, T., Smith, N. \& Kowada, A., Characterizing crustal earthquake slip models for the prediction of strong ground motion. Seismological Research Letters, 70(1), pp. 59-80, 1999.

[4] Atkinson, G. and Silva, W., Stochastic modeling of California ground motions. Bulletin of the Seismological Society of America, 90(2), pp. 255-274, 2000.

[5] Irikura, K., Prediction of strong motions from future earthquakes caused by active faults-case of the Osaka basin. Proc. of the $12^{\text {th }}$ World Conf. on Earthquake Engineering, Auckland, 2000.

[6] Tao, X.X. \& Wang, H.Y., A random source model for near field strong ground motion prediction. Proc. of the 13th World Conf. on Earthquake Engineering, Vancouver, Canada, 2004.

[7] Boatwright, J.G. \& Choy, G.L., Acceleration source spectra anticipated for large earthquakes in northeastern North America. Bulletin of the Seismological Society of America, 82(2), pp. 660-682, 1992.

[8] Beresnev, I.A. \& Atkinson, G., Generic finite-fault model for groundmotion prediction in Eastern North America. Bulletin of the Seismological Society of America, 89(3), pp. 608-625, 1999.

[9] Liu, H.M., Tao, X.X., Sun, X.D. \& Li, P., Forecast of strong ground motion field near the fault for earthquake disaster reduction in urban area. Earthquake Resistant Engineering Structures VII, eds. M. Phocas, C.A. Brebbia \& P. Komodromos, WIT Press: Southampton, pp. 271-278, 2009.

[10] Herrero, A. \& Bernard, P., A kinematic self-similar rupture progress for earthquake. Bulletin of the Seismological Society of America, 84, pp. 1216-1228, 1994.

[11] Atkinson, G. \& Boore, D.M., Evaluation of models for earthquake source spectra in eastern North America. Bulletin of the Seismological Society of America, 88(4), pp. 917-934, 1998.

[12] Beresnev, I.A. \& Atkinson, G., Modeling finite-fault radiation from the $\omega n$ spectrum. Bulletin of the Seismological Society of America, 87(1), pp. 67-84, 1997.

[13] Motazedian, D. \& Atkinson, G.M., Stochastic finite-fault modeling based on a dynamic corner frequency. Bulletin of the Seismological Society of America, 95(3), pp. 995-1010, 2005. 